\title{
LE VITTIME DELL'AMIANTO E LA CORTE DI STRASBURGO: LA TUTELA CAUTELARE QUALE POSSIBILE GIUSTIZIA RIPARATRICE *
}

\author{
LAS VÍCTIMAS DEL AMIANTO Y LA CORTE EUROPEA \\ DE DERECHOS HUMANOS: LA PROTECCIÓN CAUTELAR \\ COMO POSIBLE JUSTICIA RESTAURATIVA
}

ASBESTOS VICTIMS AND THE EUROPEAN COURT OF HUMAN RIGHTS: THE INTERIM MEASURES AS POSSIBLE RESTORATIVE JUSTICE

\section{RESUMEN}

El presente trabajo expone un análisis argumentativo en torno a la protección cautelar en general y en particular a la protección cautelar de las víctimas del amianto en el sistema de la Corte Europea de Derechos Humanos. Delimita el concepto de protección cautelar, sus características y elementos esenciales, menciona la legislación sobre el amianto vigente en el sistema jurídico italiano.

Aborda otros temas de relevancia jurídica entre los que se destacan (i) La duración excesiva del procedimiento para las víctimas del amianto en el sistema jurídico italiano. (ii) La evolución reciente

*Artículo de reflexión que corresponde a resultados de investigación terminada, elaborado desde el punto de vista crítico, protección cautelar en torno a la protección cautelar y las víctimas del amianto, en el sistema de la Corte Europea de Derechos Humanos. Este artículo es producto de una monografía de Doctorado en Derechos Humanos, Universidad La Sapienza, Roma.

a. Ph. D. en Derechos Humanos en la Universidad La Sapienza, Roma, Italia. Grado en ciencias políticas y relaciones internacionales, Universidad de La Sapienza, cum laude. Ha sido responsable del Departamento de Derechos Humanos para el centro legal de amianto y Responsable de Protección Internacional y Europeo de Derechos Humanos en la Telecommunications Industry Association (TIA). Reconocida artículista con publicaciones en en revistas científicas europeas, conferencista y periodista.

\footnotetext{
MISIÓN JURÍDICA Revista de Derecho y Ciencias Sociales Bogotá, D.C. (Colombia) Colaboradores Externos Internacionales Núm. 8, Año 2015 enero-junio, pp. 23-38. ISSN 1794-600X
} 
de la jurisprudencia italiana: sentencia del Tribunal de Casación IV^^ sez. pen., 08.05.2014, n. 18933. (iii) La evolución reciente de la jurisprudencia de la Corte Europea de Derechos Humanos: affaire Vincent Lambert.

\section{PALABRAS CLAVE}

Protección cautelar en el sistema de la Corte Europea de Derechos Humanos. Protección cautelar y víctimas del amianto en el sistema de la Corte Europea de Derechos Humanos. Víctimas del amianto. Amianto. Legislación sobre el amianto en el sistema jurídico italiano.

\section{ABSTRACT}

The present document reflects an argumentative analysis related to the interim measures in general and in particular to the interim measures for the protection of asbestos victims in the legal system of the European Court of Human Rights. It also places the limits of the interim measures, its definition, its features and or, its essential elements. Additionally, it mentions legisltation on asbestos in the Italian legal system.

It mentions relevant legal topics from where we can highlight the following:

- Excessive duration of the procedure for asbestos victims in the Italian legal system.

- Recent developments in the Italian case law: Court of Cassation IV^ ${ }^{\wedge}$ sez. pen., 08.05.2014, n. 18933.

- Recent evolution of jurisprudence in the European Court of Human Rights: affaire Vincent Lambert.

\section{KEY WORDS}

Interim measures in the European Court of Human Rights, interim measures and asbestos victims in the European Court of Human Rights, Asbestos Victims, asbestos, asbestos in the Italian legal system.

\section{SINTESI}

Questo lavoro presenta un'analisi argomentativa in merito alla tutela cautelare e alla tutela cautelare e le vittime dell'amianto nel sistema della Corte Europea dei Diritti dell'Uomo.
Viene, dapprima, definito il concetto di tutela cautelare, le sue caratteristiche e/o i suoi elementi essenziali, e, poi, si esamina la normativa vigente in materia di amianto nell'ordinamento giuridico italiano.

Si affrontano successivamente altre questioni di rilevanza giuridica fra cui: (i) La durata eccessiva dei procedimenti per le vittime dell'amianto nel sistema giuridico italiano. (ii) I recenti sviluppi giurisprudenziali nel sistema giuridico italiano: sentenza Corte di Cassazione, $\mathrm{IV}^{\wedge}$ sez. pen., 08.05.2014, n. 18933. (iii) I recenti sviluppi giurisprudenziale nel sistema giuridico della Corte europea dei Diritti dell'Uomo: affaire Vincent Lambert.

\section{PAROLE CHIAVE}

Tutela cautelare nel sistema della Corte Europea dei Diritti dell'Uomo. Tutela cautelare e vittime dell'amianto nel sistema della Corte Europea dei Diritti dell'Uomo. Vittime dell'amianto. Amianto. Normativa sull'amianto nel sistema giuridico italiano.

\section{RESUMO}

Este artigo apresenta uma análise argumentativa ao redor das medidas cautelares de proteção em geral e, em particular, de proteção às vítimas do amianto no sistema do Tribunal Europeu dos Direitos Humanos. Define o conceito de proteção cautelar, suas características e elementos essenciais, mencionando a legislação sobre o amianto existente no sistema jurídico italiano.

Aborda outras questões de relevância jurídica, entre as quais se destacam: (i) a duração excessiva do processo para as vítimas do amianto no sistema jurídico italiano; (ii) recentes desenvolvimentos na jurisprudência italiana: sentença de Tribunal de Cassação IV^ ${ }^{\wedge}$ sez. pen., 08.05.2014, n. 18933; (iii) recente evolução da jurisprudência do Tribunal Europeu dos Direitos Humanos: affaire Vincent Lambert.

\section{PALAVRAS-CHAVE}

Medidas cautelares no sistema do Tribunal Europeu dos Direitos Humanos. Medidas cautelares e vítimas do amianto no sistema do Tribunal Europeu dos Direitos Humanos. Vítimas 
do amianto. Amianto. Legislação sobre o amianto no sistema jurídico italiano.

\section{INTRODUZIONE}

Come premessa generale va detto che, la materia cautelare è una forma di intervento anticipato volto a fronteggiare il prodursi di eventi che rischierebbero di vanificare l'effettività stessa della tutela giurisdizionale ${ }^{1}$.

Essa si estrinseca nell'adozione di «provvedimenti aventi quale esigenza tipica quella di affrontare temporaneamente una situazione di urgenza ed evitare un danno irreparabile in attesa di una decisione definitiva» ${ }^{2}$.

Nell'ambito della giurisdizione europea di protezione dei diritti umani è l'art. 39 del Regolamento della Corte di Strasburgo a disciplinare il c.d. "potere cautelare" ${ }^{\text {. }}$

1 G. CHIOVENDA, Istituzioni di diritto processuale civile, Napoli, 1936, I, p. 147: "La necessità di servirsi del processo non deve tornare a danno di chi ha ragione".

2 G. CORNU (dir.),Vocabulaire juridique, 6 ed., Paris, 2004, nota 33, p. 577.

3 Cfr. Sentenze della Corte europea dei Diritti dell'Uomo del 11 luglio 2000, causa 40035/98, Jabari c. Turchia; del 10 novembre 2005, causa 8062/04, Sinan Eren c. Turchia; del 10 novembre 2005, causa 26050/04, Gurbuz c. Turchia; del 10 novembre 2005 causa 24040/04, Kuruçay c. Turchia; del 10 novembre 2005, causa 22913/04, Tekin Yildiz c. Turchia; del 10 novembre 2005, causa 7454/04, Uyan c. Turchia. Decisione del 6 ottobre 1976, causa 7317/75, Lynas c. Svizzera: Decisione della Commissione del 7 dicembre 1989, causa 15658/89, Mansi c. Svezia; Sentenza della Corte europea dei Diritti dell'Uomo del 20 marzo 1991, causa 15576/89, Cruz Varas e altri c. Svezia; Decisione della Commissione sulla ricevibilità del 16 ottobre 1992, causa 18560/91, D.S., S.N. B.T. c. Francia; del 18 ottobre 1993, causa 19776/92, Barir e Amuur c. Francia; del 23 gennaio 1997, causa 32824/96, Berke c. Francia; del 18 settembre 1997, causa 34795/97, A.B. c. Francia; Decisione del 18 maggio 1999, causa 48135/99, Bodika c. Francia; Decisione sulla ricevibilità del 3 luglio 2001, causa 44190/98, Nivette c. Francia; Sentenza della Corte europea dei Diritti dell'Uomo del 5 febbraio 2002, causa 51564/99, Conka e altri c. Belgio; Decisione sulla ricevibilità del 16 aprile 2002, causa 65964/01, Penafiel Salgado c. Spagna Sentenza della Camera del 6 febbraio 2003, causa 46827/99 e 46951/99 58, Mamatkulov e Abdurasulovic c. Turchia; Sentenze della Corte europea dei Diritti dell'Uomo del 12 aprile 2005 causa 36378/02, Chamaiev e altri c. Georgia e Russia; del 10 agosto 2006, causa 24668/03, Olaechea Cahuas c. Spagna; Decisione per l'ammissibilità del 20 febbraio 2007, causa 35865/03, Al-Moayad c. Germania; Sentenze della Corte europea dei Diritti dell'Uomo del 20 settembre 2007, causa 45223/05, Sultani c. Francia; del 24 febbraio 2009, causa 246/07, Ben Khemais c. Italia; del 22 settembre 2009, causa 30471/08, Abdolkhani et Kariminia c. Turchia; del 3 dicembre 2009, causa 19576/08, Daoudi c. Francia; del 2 marzo 2010, causa 61498/08, Al-Saadoon e Mufdhi c. Regno Unito; del 3 giugno 2010, causa 52812/07, Kamaliyevy c. Russia; del 13 luglio 2010; causa 33526/08, D. B. C. Turchia; del 1 aprile 2010, causa 24268/08, Klein C. Russia; del 13 aprile 2010, causa 50163/08, Trabelsi c.
Benché non vi sia dubbio che tale competenza sia espletata in misura maggiore nei confronti di determinate categorie di vittime ${ }^{4}$ (espulsi ${ }^{5}$, estradati ${ }^{6}$ ) si rinviene, tuttavia, la possibilità di considerare la tutela cautelare in un contesto nuovo rispetto a quelli usualmente esaminati nella giurisprudenza di Strasburgo.

Si suppone, infatti, che la condizione del lavoratore gravemente malato possa rispecchiare, sia nell'urgenza dovuta alle prospettive di vita limitate, sia nelle violazioni subite, una delle fattispecie potenzialmente idonee alla richiesta di misure provvisorie.

Nello specifico, si tende a ritenere che i danni prodotti sugli individui dall'esposizione a sostanze nocive assumano caratteristiche peculiari, tali da far ritenere l'ipotesi che si intende sostenere ammissibile.

L'analisi svolta dimostra come il sistema più favorevole all'individuo sia proprio quello di tutela cautelare in seno alla Corte di Strasburgo.

\section{METODOLOGIA}

Attraverso la disanima di uno specifico ambito normativo interno (normativa e giurisprudenza italiane) si è giunti ad evidenziare come i potenziali ricorrenti gravemente ed incurabilmente malati si trovino, a causa dell'inefficienza delle vie

Italia; del 17 gennaio 2012, causa 8139/09, Omar Othman (Abu Qatada) c. Regno Unito; del 24 gennaio 2012, causa 26494/09, Ahmed Ali c. Paesi Bassi e Grecia.

4 I casi a cui si fa riferimento sono quelli in materia di espulsione o estradizione in cui si paventi il rischio di violazione dell'art. 3 della CEDU.

5 Cfr. Sentenze della Corte europea dei Diritti dell'Uomo del 20 marzo 1991, causa 15576/89, Cruz Varas c. Svezia; del 30 ottobre 1991, causa 13163/87, Vilvarajah c. Regno Unito; del 17 dicembre 1996, causa 1996 - VI, Ahmed c. Austria; del 29 aprile 1997, causa 1997 - III, H.L.R. c. Francia; del 2 maggio 1997, causa 1997 - III, D. c. Regno Unito; del 17 febbraio 2004, causa 61350/00, Thampibillai c. Paesi Bassi; del 22 novembre 2005, causa 13284/04, Bader e Kanbor c. Svezia; del 5 luglio 2005, causa 2345/02, Said c. Olanda; del 26 luglio 2005, causa 38885/02, N. c. Finlandia; del 4 luglio 2006, causa 59450/00, Ramirez Sanchez c. Francia; del 26 aprile 2007, causa 25389/05, Gebremedhin c. Francia; del 10 aprile 2012, causa 24027/07, Babar Ahmad e altri c. Regno Unito; del 17 gennaio 2012, causa 9146/07, 32650/07, Harkins e Edwards c. Regno Unito.

6 Cfr. Sentenze della Corte europea dei Diritti dell'Uomo del 7 luglio 1989, causa 14038/88, Soering c. Regno Unito; del 12 aprile 2005, causa 36378/02, Chamaïev e altri c. Georgia e Russia; del 22 dicembre 2008, causa 46468/06, Aleksanyan c. Russia. 
di ricorso interne ${ }^{7}$, nella condizione di dover continuare a lavorare per provvedere ai loro bisogni fondamentali e a quelli dei familiari.

In casi come questi «l'inadeguatezza dei rimedi interni non aventi effetto sospensivo rispetto alle esigenze imposte dall'art. 13 CEDU determina, (...), il superamento della regola del previo esaurimento e l'esercizio da parte della Corte di una "tutela cautelare sostitutiva" rispetto a quella che avrebbe dovuto essere apprestata dalle autorità nazionali. In un siffatto contesto, l'adozione di misure provvisorie anteriormente al concludersi delle procedure interne di ricorso non deve sorprendere, in quanto avviene in presenza delle stesse circostanze che legittimerebbero l'esercizio della tutela principale e risulta, in ultima analisi, preordinata a salvaguardare la stessa operatività dell'obbligo strumentale posto dall'art. 13 CEDU» ${ }^{8}$.

Tale circostanza, aggravata dal protrarsi dell'esposizione delle presunte vittime alle sostanze nocive responsabili della malattia, tende a concretizzare una violazione da parte dello Stato degli articoli 2, 3 e 8 della Convenzione e a creare i presupposti per l'invocazione dell'adozione di misure provvisorie data la natura "grave ed irreparabile" del pregiudizio subito dai ricorrenti.

Da un lato, infatti, l'aspetto sostanziale viene "pregiudicato" con la violazione del diritto alla salute, alla dignità umana e alla vita (artt. 2, 3, 8 della Convenzione), dall'altro, quello procedurale viene "compromesso" con la morte dei ricorrenti ${ }^{9}$ (art. 34 della Convenzione).

7 Cfr. La condizione di numerosi lavoratori esposti all'amianto. Rif. Sentenza della Corte di Cassazione IV Sez. Pen., del 2008, causa 42128

8 A. SACCUCCI, "Le misure provvisorie nella protezione internazionale dei diritti umani", Torino, 2006, pp. 258-259.

9 La questione del venir meno dell'oggetto del contenzioso è stata affrontata in questi termini dalla giurisprudenza della Corte già in casi di adozione di misure provvisorie allo scopo di porre fine a pratiche di autolesionismo. Cfr. a tal proposito Sentenza della Corte europea dei Diritti dell'Uomo del 8 luglio 2004, causa 48787/99, Ilascu e altri c. Moldavia e Russia, \$ 11. Inoltre nella sentenza si legge che: $i$ Governi convenuti dovranno "prendre toutes les mesures nécessaires afin d'assurer (au requérant), en grève de la faim depuis (une quinzaine de jours), des conditions de détention conformes au respect de ses droits garantis par la Convention".

\section{RISULTATI}

L'adozione della misura provvisoria tende pertanto a creare una temporanea condizione di status quo che permette alle vittime di salvaguardare il loro stato di salute e conseguentemente il loro diritto alla vita.

\section{POTENZIALI RICORRENTI}

$\mathrm{Si}$ ritiene possibile attribuire la facoltà di proporre questo tipo di ricorsi ad individui che abbiano contratto "patologie da esposizione ad agenti cancerogeni", nello specifico, "lavoratori esposti all'amianto".

Prima di passare ad un'analisi più dettagliata della questione è bene precisare che l'ipotesi qui considerata non presuppone comunque l'inidoneità di altri individui vittime di violazioni simili a quelle enunciate ad avanzare ricorso (militari impiegati in aree con presenza di uranio impoverito, individui esposti a fumi tossici...)

Rispetto alla categoria di ricorrenti oggetto di studio sembra anzitutto utile evidenziare alcuni aspetti.

Non vi è dubbio che la prima cosa che salti agli occhi sia la numerosità dei soggetti lesi tanto da non sembrare improprio parlare di "danni di massa".

A questa prima questione seguono la responsabilità dello Stato, la rilevanza delle misure di prevenzione e del controllo pubblico e la lungolatenza del danno.

Un altro elemento da considerare sembrerebbe essere quello della sussistenza di un nesso causale fra la condotta colposa dello Stato ed il danno patito a seguito dell'esposizione a sostanze nocive. La ricostruzione di tale legame sarebbe individuabile nel cosiddetto "effetto patogeno" prodotto dalle sostanze e, cioè, nell'esposizione interna del corpo umano (inalazione ed ingestione) alle radiazioni emesse dalle polveri di amianto, nonché alla tossicità chimico fisica delle stesse $^{10}$ da cui conseguirebbe la

10 Cfr. Air Force Armament Laboratory, Morphological Characteristics of Particulate material Formed from High Velocity Impact of Depleted Uranium Projectiles with Armor Targets. Final Report for period October 1977 - October 1978 IARC, Monograph vo. 14, "Asbetos", 1977; Gustavsson et al. (1998) "Occupational exposures and squamous cell carinoma of 
responsabilità dello Stato configurabile nella mancata ottemperanza alla dovuta e necessaria adozione di opportune misure di sicurezza e prevenzione.

Richiamate tali essenziali premesse si procederà di seguito ad un esame più approfondito della questione che tenterà di valutare, sulla base della giurisprudenza e della prassi, la concreta possibilità per questi individui di poter avanzare una richiesta di misure provvisorie al fine di tutelare il loro diritto alla salute e alla vita.

\section{I possibili diritti da invocare e la questione dell'ambito di applicazione}

Come già accennato, la facoltà per la categoria di vittime individuate di proporre questo tipo di ricorsi verrebbe loro attribuita dalla natura stessa della pretesa violazione.

Trattandosi, infatti, di "danni gravi ed irreparabili" alla salute e alla vita si tenderebbe ad ipotizzare una violazione degli artt. 2 e 3 (diritto alla vita, divieto ai trattamenti inumani e degradanti) in combinato con l'art. 8 (diritto alla vita privata e familiare) da cui potrebbe derivare una violazione dell'art. 34 (diritto al ricorso individuale ${ }^{11}$.

Risultano pertanto "compromessi" sia gli "interessi delle parti" che l' "oggetto della procedura”.

A questo proposito è opportuno evidenziare che l'art. 39 del Regolamento della Corte preveda al primo comma che l'adozione di misure provvisorie debba avvenire "nell'interesse

the oral cavity pharinx, larynx and oesophagus: a case - control study in Sweden", Occup. Environ med. 55: 393 - 400; Governa M., Amati M., Fontana S. et al., Role of the iron in absestos - bodyinduced oxidant radical generation, J. Toxicol Environ Healt part A 1999; 58: 279 - 87.

11 A. SACCUCCI, "Le misure provvisorie nella protezione internazionale dei diritti umani", Torino, 2006, p. 346: "Ancorando la nozione di "pregiudizio grave ed irreparabile" all'incidenza che la violazione di determinati diritti può avere sulla vita o sull'integrità psico - fisica della persona umana si riesce, dunque, a fornire, una chiave di lettura abbastanza unitaria e coerente della più gran parte della giurisprudenza degli organi internazionali di protezione dei diritti umani e si precostituisce, al contempo, una valida premessa per l'emergere di orientamenti evolutivi volti ad estendere l'ambito di esplicazione del rimedio cautelare tanto in relazione a nuovi pericula incidenti su tali beni quanto in base ad un più ampio apprezzamento degli elementi che li compongono e ciò vale soprattutto per gli aspetti dell'integrità psicologico - morale della persona umana)." delle parti o della corretta conduzione del procedimento" e che il paragrafo 104 della sentenza Mamaktulov ${ }^{12}$ individui quale ambito di applicazione della tutela cautelare gli artt. 2, 3 e 8 della CEDU ${ }^{13}$.

Verranno ora approfonditi ed esaminati tutti gli aspetti che tendono a rendere legittima l'invocazione delle disposizioni qui richiamate nei casi oggetto di studio.

\section{L'eccesiva durata dei procedimenti in termini di riconoscimento dei benefici contributivi e l'esigenza di una tutela cautelare}

L'eccessiva durata dei procedimenti è ciò che in termini assoluti affermi con più vigore e legittimi l'adozione in questo ambito di misure provvisorie.

I tempi estremamente lunghi per giungere ad una sentenza definitiva, infatti, annullano e vanificano il diritto.

«Le vittime vedono riconosciuti i loro diritti contributivi quando hanno già raggiunto l'età pensionabile o la massima anzianità contributiva o addirittura, cosa ancor più grave, quando sono già ammalati o deceduti, per l'esposizione all'amianto» ${ }^{14}$ (o ad altre sostanze cancerogene).

Viepiù che, trattandosi di lavoratori, c'è anche da considerare l'ipotesi che questi, per non correre il rischio di rimanere privi di reddito, continuino a svolgere la loro attività lavorativa in esposizione a sostanze che, anche

12 Cfr. Sentenza della Corte europea dei Diritti dell'Uomo, del 6 febbraio 2003, causa 46827/99 e 46951/99 58, Mamatkulov e Abdurasulovic c. Turchia.

13 En l'espèce, la situation que vous exposez dans votre courrier ne correspond pas à celles dans lesquelles la mesure prévue à l'article 39 peut être appliquée. L'indication de mesures provisoires ne s'exerce que dans des domaines limités. Sans doute reçoit-elle un certain nombre de demandes, mais en principe ce n'est que lorsqu'il y a risque de dommage irréparable et que le risque est imminent que la Cour applique l'article 39. Bien qu'il n'existe pas de disposition particulière dans la Convention concernant ces domaines, les demandes ont trait le plus souvent au droit à la vie (article 2), au droit de ne pas être soumis à la torture et aux traitements inhumains (article 3) et exceptionnellement au droit au respect de la vie privée et familiale (article 8) ou à d'autres droits garantis par la Convention. La grande majorité des mesures provisoires ont été indiquées dans des affaires d'expulsion et d'extradition"

14 E. BONANNI, "Lo Stato dimentica l'amianto killer. Diritti negati. Ricorso a Strasburgo", Firenze, 2009, pp. 133; 142 - 144 
«a basse dosi e sporadicamente, abbreviano enormemente i tempi di latenza e moltiplicano il rischio di malattie professionali in immanente contrasto con le norme costituzionali e con i principi dell'ordinamento giuridico interno ed internazionale» ${ }^{15}$.

\section{La normativa italiana in tema di amianto}

Nel 1931 la legislazione inglese fu la prima ad affermare l'esigenza di un controllo delle polveri di amianto nel settore industriale e a riconoscere l'importanza di un risarcimento dei danni.

Nello stesso anno Klemperer e Rabin coniarono il termine "mesotelioma". Iniziò così a prendere vigore l'ipotesi di un potenziale rapporto di causalità fra l'esposizione all'amianto e l'enorme incidenza di tumore al polmone ${ }^{16}$.

Oltre all'Inghilterra gli studi epidemiologici interessarono l'Italia, il Sud Africa e l'America.

Vennero individuati quali principali fattori del potenziale rischio morbigeno tre parametri: dose di esposizione, durata dell'esposizione e dimensione delle fibre.

Molteplici furono le patologie ritenute "asbesto17 correlate"18: pleuropatie benigne, asbestosi polmonare, carcinoma polmonare e mesotelioma ${ }^{19}$.

In Italia l' "asbestosi" venne riconosciuta quale malattia professionale con la legge 455 del 1943.

15 E. BONANNI, "Lo Stato dimentica l'amianto killer. Diritti negati. Ricorso a Strasburgo", Firenze, 2009, p. 128. Ne ha dato conferma anche la Corte di Cassazione: Sentenza della Corte di Cassazione, $I V^{\wedge}$ sez. pen., 08.05.2014, n. 18933.

16 E. BONANNI, "Lo Stato dimentica l'amianto killer. Diritti negati. Ricorso a Strasburgo", Firenze, 2009, p. 74.

17 Il termine fu coniato nel 1927 da W. E. Cooke. Con esso si definiva: "quella strana fibrosi polmonare che colpiva i lavoratori dell'amianto." Nel 1966 lo studioso inglese Merewether scoprì che il 66\% di lavoratori a tempo indeterminato dell'industria Rochdale ne era affetto. E. BONANNI, "Lo Stato dimentica l'amianto killer. Diritti negati. Ricorso a Strasburgo", Firenze, 2009, p. 74.

18 GUSTAVSSON et al. (1998) "Occupational exposures and squamous cell carinoma of the oral cavity, pharinx, larynx and oesophagus: a case - control study in Sweden", Occup. Environ med. 55: 393 - 400 .

19 E. BONANNI, "Lo Stato dimentica l'amianto killer. Diritti negati. Ricorso a Strasburgo", Firenze, 2009, pp. 78 - 79: Soffritti (2004) sosteneva: "Oltre al mesotelioma, l'amianto determina un aumento dell'incidenza di altri tumori, in particolare di quelli del polmone, della laringe, dell'esofago, del colon - retto e del rene".
Negli anni a seguire vari decreti riproposero tale legge non apportandovi alcuna modifica sostanziale $^{20}$

Il primo intervento concreto da parte del legislatore italiano giunse solo nel 1991 con il D. Lgs. 277 del $1991^{21}$ che recepì la Direttiva Comunitaria 477/83/CEE ${ }^{22}$.

Il 27.03.1992 $2^{23}$ venne poi approvata la Legge 257: «Norme relative alla cessazione dell'impiego dell'amianto». In realtà il titolo della normativa può indurre a formulare l'ipotesi di un'avvenuta presa di coscienza relativamente alla questione in oggetto ma l'articolo 1 smentisce qualsivoglia incoraggiante supposizione disponendo che: «sono vietate (soltanto) l'estrazione, l'importazione, l'esportazione, la commercializzazione e la produzione di amianto, di prodotti di amianto o prodotti contenenti amianto» ${ }^{24}$

Nel frattempo, nella giurisprudenza ${ }^{25}$ emerse con forza la volontà di ridurre la portata

20 Cfr. DPR 648/1956; DPR 1124/1965. Cfr. BONANNI,"Lo Stato dimentica l'amianto killer. Diritti negati. Ricorso a Strasburgo", Firenze, 2009, p. 93.

21 Vengono introdotte per il riconoscimento dei benefici contributivi delle soglie di esposizione: 100 fibre/litro sulla media di otto ore lavorative per oltre 10 anni. In realtà tali limiti sono avversati da una nutrita giurisprudenza: "Anche basse concentrazioni e sporadiche esposizioni hanno un ruolo acceleratore...e conseguente rilevanza causale, connessa alla abbreviazione della latenza e all'anticipazione dell'evento letale (Cfr. Cassazione Sezione Penale, sentenza n. 42128 del 12.11.2008). Cfr. inoltre Cassazione 21682/2004: Cassazione Sezione Lavoro 16119/2005. Cassazione 16119/2005: Relazione Giudice del Lavoro Tribunale di Ravenna Dott. Roberto Riverso per incontro CSM sulla formazione dei magistrati del 26.11.2008 "Il rischio amianto: le controversie in materia di maggiorazione contributiva".

22 Cfr. Direttiva sulla protezione dei lavoratori contro i rischi connessi con l'esposizione all'amianto durante il lavoro (II direttiva particolare ai sensi dell'art. 8 della direttiva 80/1107/ CEE)

23 Cfr. Gazzetta Ufficiale n. 87 del 13/4/1992.

24 E. BONANNI,"Lo Stato dimentica l'amianto killer. Diritti negati. Ricorso a Strasburgo", Firenze, 2009, p. 108.

25 Cfr. Sentenze Cassazione Sezione Lavoro n.21256/2004; 2456/2005: 2587/2005; 1392/2005, 2582 - 83 85/2005; 16117/2005. Sentenze del Tribunale di Terni confermate in Cassazione: 22432/2004; 22433/2004; 22435/2004; 22446/2004; 377/2004; 2243/2004; 22519/2004; 22541/2004; $22831 / 2004$ : 23524/2004: 21929 - 2031 - 32/2004 - 21889 90 - 91/2004; 1980/2004; 4557/2004; 4550/2004; 2415/2004 1969/2004; 4665/2004; 3463/2004; 2416/2004; 2408/2004; 2475/2004; 1974/2004; 22313 - 14 - 15 - 16 - 17 - 18/2004 22300/2004; 21864 - 65 - 66 - 67 - 68/2004: 396/2004 $320 / 2004$; 22156 - 57 - 58/2004: 21861/2004: 21991/2004; 22301 - 02 - 03 - 04/2004; 22079 - 80/2004; 20465/2004; 10673/2004; 21223/2004; 21330 - 31/2004; 21445/2004. 
del parametro delle soglie di esposizione, introdotto con il D. Lgs. 277 del 1991, a favore di un accertamento di tipo presuntivo fondato sulla verosimiglianza e sull'analogia ${ }^{26}$ : «al fine del riconoscimento di tale beneficio, non è necessario che il lavoratore fornisca la prova atta a quantificare con esattezza la frequenza e la durata dell'esposizione, potendo ritenersi sufficiente, qualora ciò non sia possibile, avuto riguardo al tempo trascorso e al mutamento delle condizioni di lavoro, che si accerti, anche a mezzo di consulenza tecnica, la rilevante probabilità di esposizione del lavoratore al rischio morbigeno, attraverso un giudizio di pericolosità dell'ambiente di lavoro, con un margine di approssimazione di ampiezza tale da indicare la presenza di un rilevante grado di probabilità di superamento della soglia massima di tollerabilità» ${ }^{27}$.

Il 30 settembre 2003 fu introdotto il Decreto Legge n. $169^{28}$ convertito con Legge 326 del 2003 che ridusse «drasticamente i benefici contributivi per esposizione all'amianto la cui maggiorazione contributiva è limitata al $25 \%$ ai soli fini della determinazione dell'importo delle prestazioni pensionistiche e non della maturazione del diritto di accesso alle medesime» e che introdusse «espressamente nella fattispecie legale il limite di soglia che prima non esisteva» ${ }^{29}$.

Fu poi emanato il 27 ottobre 2004, in attuazione dell'art. 47 comma VI del D.L. n. 266/03, il Decreto del Ministero del Lavoro e delle Politiche Sociali.

Furono individuati attraverso 500 atti di indirizzo i siti industriali dove maggiore e più grave potesse essere il rischio per i lavoratori e dove fosse comprovato il superamento della soglia di esposizione.

Tali provvedimenti furono successivi alla condanna che l'Italia subì nella Sentenza del 15 novembre 2001 da parte della Corte di Giustizia Europea per «l'inadempimento di direttive comunitarie relative alla sicurezza e all'integrità

26 E. BONANNI,"Lo Stato dimentica l'amianto killer. Diritti negati. Ricorso a Strasburgo", Firenze, 2009, p. 112.

27 Cfr. Sentenza della Cassazione Sezione Lavoro n. 16119/2005.

28 Pubblicato in G.U. del 2 ottobre 2003, n. 229.

29 E. BONANNI,"Lo Stato dimentica l'amianto killer. Diritti negati. Ricorso a Strasburgo", Firenze, 2009, p. 115 fisica e per la mancata osservanza delle Direttive Comunitarie 89/391/CEE e 473/83/CEE».

La necessaria ed obbligata rivisitazione del complesso normativo condusse all'approvazione definitiva del D. Lgs. n. 81 del $2008^{30}$ in attuazione delle direttive comunitarie ${ }^{31}$ in tema di tutela della salute e di sicurezza nei luoghi di lavoro.

Il presente Decreto riordinò la complessa normativa e dedicò il capo III alla "protezione dai rischi connessi all'esposizione all'amianto» ${ }^{32}$.

Tuttavia, la genericità delle disposizioni e il linguaggio «tecnicamente scadente e discorsivo» ${ }^{33}$ diedero luogo all'attuale quadro normativo ancora molto lontano dagli standard di protezione comunitario ed internazionale.

Fra le problematiche emergenti continuò ad esservi quella, di non trascurabile importanza, dell'eccessiva durata dei procedimenti in termini di riconoscimento dei benefici contributivi le cui gravi conseguenze hanno spinto a considerare come necessario in tale ambito il ricorso alla tutela cautelare.

I più recenti sviluppi giurisprudenziali interni ed europei e la possibile applicazione della tutela cautelare all'ambito considerato

Corte di Cassazione, IV^ ${ }^{\wedge}$ sez. pen., 08.05.2014, n. 18933: la sentenza che "blinda" la presentazione di ricorso ex art.39 in materia di amianto

La recentissima sentenza della Corte di Cassazione in materia di amianto ${ }^{34}$ rafforza in modo inequivocabile quanto si sta tentando di sostenere.

L'argomento è del massimo interesse poiché consente di affermare ulteriormente ed in maniera certa la possibilità per gli individui

30 Cfr. Del 9 aprile 2008 pubblicato nella Gazzetta Ufficiale $n$. 101 del 30 aprile 2008.

31 Cfr. 89/391/CEE, 89/654/CEE, 90/269/CEE, 90/270/CEE, 90/394/CEE, 90/679/CEE, 93/88/CEE, 95/63/CE, 97/42/CE, 98/24/CE, 99/38/CE, 99/92/CE, 2001/45/CE, 2003/10/CE, 2003/18/CE, 2004/40/CE.

32 Cfr. La nuova normativa è contemplata negli artt. $246 e$ seguenti del Decreto.

33 E. BONANNI,"Lo Stato dimentica l'amianto killer. Diritti negati. Ricorso a Strasburgo", Firenze, 2009, p. 120.

34 Cfr. Corte di Cassazione, IV ${ }^{\wedge}$ sez. pen., del 08.05.2014, $n$. 18933. 
"vittime da esposizione" di poter presentare un ricorso ex art. 39.

Nella presente pronuncia la Corte riconosce che: « (...) ritenere acquisita la indicata stadiazione irreversibile, non implica affatto che la prosecuzione dell'esposizione non produca una accelerazione dei tempi della progressione della patologia. Insomma, per il ricorrente, irreversibilità non significa che si è esclusa la ulteriore attività di promozione esercitata dalla protratta esposizione all'agente cancerogeno. (...) L'amianto, alla luce delle evidenze statistiche, costituisce sicuro fattore di iniziazione. Ma non vi sono solide evidenze che contrastino l'ipotesi che esso sia pure promotore: vi è plausibilità biologica, giacché la flogosi cronica potrebbe fornire un importante stimolo alla proliferazione di cellule già iniziate al processo di cancerogenesi. (...) Non esiste esposizione irrilevante. (...) Inoltre, costituisce sapere scientifico condiviso il fatto che l'evidenza epidemiologica disponibile sia univoca nell'indicare una relazione proporzionale tra dose cumulativa ed incidenza, nel senso che all'aumento dell'esposizione per intensità e durata aumentano i casi di tumore all'interno della popolazione esposta. Ancora, l'orientamento prevalente della giurisprudenza di legittimità è indirizzato nel senso della rilevanza dell'effetto acceleratore. (...)».

In altre parole, con tale rivoluzionaria decisione la Corte non soltanto ha fornito nuovi ed ulteriori strumenti in materia di attribuzione di responsabilità penale e civile, ma ha affermato, con chiarezza senza precedenti, che: «la protratta esposizione all'amianto crea un'ulteriore attività di promozione che produce una accelerazione nei tempi di progressione della patologia».

Ciò costituisce la base essenziale per affermare con certezza che l'adozione di misure provvisorie in un simile ambito non possa che essere indispensabile. L'immediato allontanamento del ricorrente dal sito nocivo è in grado di rallentare il decorso della malattia.

La celerità del procedimento, pertanto, non consentirebbe solo, come è stato già precedentemente chiarito, il riconoscimento dei benefici contributivi, ma produrrebbe un risultato di assoluta e maggiore rilevanza e cioè la garanzia per il ricorrente di un'aspettativa di vita maggiore.

\section{L'affaire Vincent Lambert}

Rispetto alla questione che si sta trattando assume rilevanza determinante una recentissima pronuncia della Corte di Strasburgo che inaugura una fase importante nel percorso evolutivo della materia cautelare.

Al di là di aspetti ulteriori che il caso potrebbe sollevare e che potranno essere oggetto di una futura trattazione, ciò che qui preme rilevare è l'orientamento giurisprudenziale della Corte con riferimento al ruolo svolto dalle misure provvisorie nell'ambito del diritto alla salute e nel contesto più generale del sistema di protezione dei diritti umani.

Per quanto riguarda il primo aspetto a rilevare è anzitutto la scelta operata dalla Corte in termini di "destinatario del provvedimento" o "vittima potenziale".

Non si tratta, infatti, né, delle più note categorie di vittime (detenuti, espulsi, estradati) tutelate dalla Corte in ambito cautelare rispetto a violazioni dell'art. 3, e, né, di una fra quelle particolari categorie di vittime (anziani, donne, bambini) che godono di specifiche misure di protezione.

Il signor Lambert è "semplicemente" un uomo di 38 anni tetraplegico in stato vegetativo da sei anni.

L'attenzione che la Corte porta a tale vicenda conferma inequivocabilmente quanto si è andato sostenendo.

A ben vedere, infatti, diviene sempre più concreta per le "vittime dell'amianto" la possibilità di avvalersi di un rimedio effettivo in grado di garantire loro un immediato ristoro per il danno subito.

Ma v'è di più, poiché a questo aspetto, si somma l'indiscutibile "ruolo evolutivo" svolto dall'art. 3 quale disposizione invocabile a "vari livelli" dalle potenziali vittime per tutte quelle violazioni che riguardino il "diritto alla salute".

Basti dire che ne le "griefs" del caso in oggetto si può leggere: "Citant l'article 3 de la Convention, ils estiment que la privation de nourriture et d'hydratation est un mauvais traitement constitutif de torture. Ils font également valoir que la privation de kinésithérapie depuis octobre 
2012 ainsi que de rééducation équivaut à un traitement inhumain et dégradant» ${ }^{35}$ e che la Corte «saisie d'une demande d'article 39 de son règlement» ha deciso di : «faire suspendre l'exécution de l'arrêt rendu par le Conseil d'État pour la durée de la procédure devant elle. La chambre a précisé que cette mesure provisoire implique que $M$. Vincent Lambert ne soit pas déplacé avec le but d'interrompre le maintien de son alimentation et de son hydratation». ${ }^{36}$

Tale emergente sensibilità non avalla solo quanto teorizzato in termini di tutela cautelare ed amianto ma anche quanto sostenuto rispetto al ruolo svolto dai provvedimenti cautelari nell'ambito del sistema europeo di protezione dei diritti umani ${ }^{37}$.

Si ritiene, infatti, che la Corte abbia da sempre riconosciuto alle misure provvisorie un ruolo di indiscutibile valore: «la Cour souligne que dans des affaires où le requérant allègue de manière plausible un risque de dommage irréparable quant à la jouissance de l'un des droits qui relèvent du noyau dur des droits protégés par la Convention, une mesure provisoire a pour objet de préserver et protéger les droits et intérêts des parties à un litige pendant devant la Cour dans l'attente de la décision finale de celle-ci» ${ }^{38}$.

L'affermazione della materia cautelare, come dimostrato dalla giurisprudenza, ha reso il sistema di protezione dei diritti umani più effettivo ed efficace cosa questa che spiegherebbe anche l'elaborazione da parte della Corte di un articolo intitolato alle misure provvisorie al fine di colmare la grave lacuna prodotta dall'assenza di una disposizione convenzionale.

La Corte ha precisato che: «une indication de mesures provisoires donnée par la Cour permet à celle-ci non seulement d'examiner efficacement une requête mais aussi de s'assurer de l'effectivité de la protection prévue par la Convention à l'égard du requérant» ${ }^{39}$.

35 Cfr. Corte Europea dei Diritti dell'Uomo, del 23 giugno 2014, causa n. 46043/14, Pierre Lambert e altri c. Francia.

36 Cfr. Note d'information sur la jurisprudence de la Cour, n. 176, luglio 2014

37 Cfr. C. QUATTROCCHI, tesi dottorale, "Le misure provvisorie secondo la Cedu e nella giurisprudenza della Corte di Strasburgo".

38 Cfr. Sentenze della Corte europea dei Diritti dell'Uomo del 10 marzo 2009, causa 39806/05, Paladi c. Moldavia, § 89.

39 Cfr. Sentenza della Corte europea dei Diritti dell'Uomo, op. cit.,
L'uso delle congiunzioni correlative non seulement...mais aussi, riconoscendo alla "corretta conduzione del procedimento" e all' "interesse delle parti" un rapporto di pari ordinazione, confermato anche in altre sentenze $\mathrm{e}^{40}$, permette di avanzare l'ipotesi che i due caratteri né si escludano a vicenda né si sovrastino.

Non sarebbe un caso, pertanto, che la «garanzia giurisdizionale di carattere preventivo» sia stata considerata uno «degli aspetti più gratificanti dell'azione di protezione internazionale dei diritti fondamentali della persona ${ }^{41}$.

La formulazione stessa dell'art. 39, come già ricordato, è da interpretarsi come la manifestazione concreta di un'ottica garantista.

La norma dispone, infatti, al I comma che: «le misure cautelari possono essere adottate nell'interesse delle parti o della corretta conduzione del procedimento».

$\mathrm{Si}$ tende a sostenere che l'articolazione della disposizione in oggetto non sia casuale e che il motivo per il quale l' "interesse delle parti" preceda la "corretta conduzione del procedimento" si possa ricondurre alla volontà della Corte di conferire un diversificato valore alle due finalità.

È possibile che, pur di rendere "efficace" e "concreta" la realizzazione dell' "interesse delle parti", la Corte si sia servita dello strumento procedurale e non viceversa, e che, sempre per lo stesso motivo, abbia scelto di far corrispondere l'inosservanza di misure provvisorie con la violazione dell'art. 34 della Convenzione.

Con tali "stratagemmi" essa sarebbe stata in grado di trasferire "per riflesso" alla materia

Chamaïev e altri c. Georgia e Russia, $\$ 473$.

40 Cfr. Sentenze della Corte europea dei Diritti dell'Uomo del 6 febbraio 2003, causa 46827/99, 46951/99, Mamatkulov e Abdurasulovic c. Turchia, \& 125; del 28 marzo 2008, causa 44009/05, Schtukaturov c. Russia, \$ 104; del 24 febbraio 2009, causa 246/07 Ben Khemais c. Italia, \$ 82; del 10 marzo 2009, causa 39806/05, Paladi c. Moldavia, \$ 86: :."(...) dans le système de la Convention, les mesures provisoires telles qu'elles ont été constamment appliquées en pratique (...) se révèlent d'une importance fondamentale pour éviter des situations irréversibles qui empêcheraient la Cour de procéder dans de bonnes conditions à un examen de la requête et, le cas échéant, d'assurer au requérant la jouissance pratique et effective du droit protégé par la Convention qu'il invoque".

41 CANCADO TRINDADE, "Les mesures provisoires devant la Cour interaméricaine des droits de l'homme », op. cit., p. 145 
cautelare l'importanza che da sempre viene attribuita nel sistema convenzionale e, che è desumibile anche dalla giurisprudenza, all'oggetto della procedura.

È proprio nell'ottica di tale impostazione che si inserisce la tutela offerta dalla Corte al Signor Vincent Lambert quale espressione della più recente evoluzione del sistema di protezione europeo dei diritti umani.

Anche in questo caso la giurisprudenza si presta con favore ${ }^{42}$ a dimostrare come la relazione fra elemento sostanziale e procedurale non impedisca di agire "concretamente" in situazioni ${ }^{43}$ che rischierebbero altrimenti di pregiudicare la posizione della "presunta vittima".

Non sembra pertanto improprio affermare che l'importanza di uno strumento di prevenzione o cessazione della potenziale violazione dei

42 Cfr. Corte Europea dei Diritti dell'Uomo, del 23 giugno 2014, causa n. 46043/14, Pierre Lambert e altri c. Francia

43 Cfr. Sentenze della Corte europea dei Diritti dell'Uomo, op. cit., Mamatkulov et Askarov c. Turchia; del 11 luglio 2000, causa 40035/98, Jabari c. Turchia. del 10 novembre 2005, causa 8062/04, Sinan Eren c. Turchia; del 10 novembre 2005, causa 26050/04, Gurbuz c. Turchia; del 10 novembre 2005, causa 24040/04, Kuruçay c. Turchia; del 10 novembre 2005, causa 22913/04, Tekin Yildiz c. Turchia; del 10 novembre 2005, causa 7454/04, Uyan c. Turchia. Decisione del 6 ottobre 1976, causa $7317 / 75$, Lynas c. Svizzera; Decisione della Commissione del 7 dicembre 1989, causa 15658/89, Mansi c. Svezia; Sentenza della Corte europea dei Diritti dell'Uomo del 20 marzo 1991, causa 15576/89 Cruz Varas e altri c Svezia. Decisione della Commissione sulla ricevibilità del 16 ottobre 1992, causa 18560/91, D.S., S.N. B.T. c. Francia; del 18 ottobre 1993, causa 19776/92, Barir e Amuur c. Francia; del 23 gennaio 1997, causa 32824/96, Berke c. Francia; del 18 settembre 1997, causa 34795/97, A.B. c. Francia; Decisione del 18 maggio 1999, causa 48135/99, Bodika c. Francia; Decisione sulla ricevibilità del 3 luglio 2001, causa 44190/98, Nivette c. Francia; Sentenza della Corte europea dei Diritti dell'Uomo del 5 febbraio 2002, causa 51564/99, Conka e altri c. Belgio; Decisione sulla ricevibilità del 16 aprile 2002, causa 65964/01, Penafiel Salgado c. Spagna; Sentenza della Camera del 6 febbraio 2003, causa 46827/99 e 46951/99 58, Mamatkulov e Abdurasulovic c. Turchia: Sentenze della Corte europea dei Diritti dell'Uomo del 12 aprile 2005, causa 36378/02, Chamaiev e altri c. Georgia e Russia; del 10 agosto 2006, causa 24668/03, Olaechea Cahuas c. Spagna; Decisione per l'ammissibilità del 20 febbraio 2007, causa 35865/03, Al-Moayad c. Germania; Sentenze della Corte europea dei Diritti dell'Uomo del 20 settembre 2007, causa 45223/05, Sultani c. Francia; del 24 febbraio 2009, causa 246/07, Ben Khemais c. Italia; del 22 settembre 2009, causa 30471/08, Abdolkhani et Kariminia c. Turchia; del 3 dicembre 2009, causa 19576/08, Daoudi c. Francia; del 2 marzo 2010, causa 61498/08, Al-Saadoon e Mufdhi c. Regno Unito; del 3 giugno 2010, causa 52812/07, Kamaliyevy c. Russia; del 13 luglio 2010; causa 33526/08, D. B. C. Turchia; del 1 aprile 2010, causa 24268/08, Klein c. Russia; del 13 aprile 2010, causa 50163/08, Trabelsi c. Italia; Casi pendenti, causa 8139/09, Omar Othman (Abu Qatada) c. Regno Unito; causa 26494/09, Ahmed Ali c. Paesi Bassi e Grecia. diritti fondamentali ${ }^{44}$, non debba risiedere tanto nella finalità teorica da esso perseguita quanto piuttosto nella sua capacità di porre in essere azioni reali e concrete di tutela degli individui.

\section{TERMINI E MOTIVAZIONI DELLE PROCEDURE}

La procedura di richiesta di adozione di misure provvisorie delle singole posizioni dovrebbe essere attivata, come da consuetudine, a domanda degli interessati.

L'adozione della misura provvisoria non tenderebbe in alcun modo a sostituire la pronuncia del tribunale nazionale ma avrebbe quale unico scopo quello di riconoscere, in via preventiva e temporanea, il beneficio del trattamento pensionistico anticipato a quei ricorrenti a cui siano stati diagnosticati tempi brevissimi di vita nonché l'allontanamento immediato dal "luogo di esposizione".

Ciò tenderebbe a sanare una grave lacuna del sistema che, ad oggi, stenta a riconoscere al ricorrente malato diritti fondamentali. Numerosi sono i casi in cui «quando finalmente si giunge alla decisione definitiva, la Sentenza semplicemente non serve più, perché l'avente diritto ha già raggiunto l'età pensionabile o la massima anzianità contributiva 0 , cosa ancora più grave, è ammalato o deceduto per l'esposizione all'amianto» ${ }^{45}$. «Nelle more questi lavoratori per non correre il rischio di rimanere privi di reddito hanno continuato a lavorare in esposizione all'amianto che, anche in basse dosi e sporadicamente, ancor più per chi è stato per decenni, già esposto, e a dosi massicce, abbrevia enormemente i tempi di latenza e moltiplica il rischio di malattie professionali, in immanente contrasto con le norme costituzionali e con i principi dell'ordinamento giuridico interno ed internazionale» ${ }^{46}$.

La misura provvisoria, pertanto, in linea con il principio del "rispetto fra giurisdizioni", non interferirebbe in alcun modo con il procedimento

44 COHEN - JONATHAN, "De l'effet juridique des " mesures provisoires " dans certaines circonstances et de l'efficacité du droit de recours individuel : à propos de l'arrêt de la Cour de Strasbourg Cruz Varas du 20 mars 1991 », R.U.D.H., 1991, p. 206 45 E. BONANNI,"Lo Stato dimentica l'amianto killer. Diritti negati. Ricorso a Strasburgo", Firenze, 2009, p. 143.

46 E. BONANNI, "Lo Stato dimentica l'amianto killer. Diritti negati. Ricorso a Strasburgo", Firenze, 2009, p. 128. 
pendente dinanzi al Tribunale nazionale, ma, piuttosto, si limiterebbe a riconoscere al ricorrente gravemente malato un temporaneo ma necessario "ristoro" (maggiori prospettive di vita, benefici economici).

Essa inoltre "sanerebbe" questo tipo di ricorsi, che, a differenza di quanto precisato nella Convenzione, si presentano "in - efficaci" e "in - effettivi" 47

Tutto ciò avrebbe come risultato la «realizzazione di una maggiore protezione dei diritti umani» ${ }^{48}$.

Avuto riguardo delle motivazioni sopra esposte è ora opportuno approfondire la questione delle violazioni invocabili dai potenziali ricorrenti.

\section{LE VIOLAZIONI INVOCABILI}

\section{L'art. 2: il diritto alla vita ${ }^{49}$}

Il diritto alla vita è un diritto fondamentale ${ }^{50}$ e figura fra quelle disposizioni la cui potenziale violazione è stata ritenuta dalla Corte come idonea all'avanzamento di richiesta di misure provvisorie. Esso impone allo Stato non solo obblighi di "non facere" ma anche obblighi positivi che dovrebbero estrinsecarsi nell'emanazione di una legislazione idonea a reprimere ogni attentato alla vita ${ }^{51}$, nella protezione della vita

47 C. ZANGHI', 'La protezione internazionale dei diritti dell'uomo", Torino, 2006, p. 250.

48 C. ZANGHI', "La protezione internazionale dei diritti dell'uomo", Torino, 2006, p. 247.

49 Art. 2: "1. Il diritto alla vita di ogni persona è protetto dalla legge. Nessuno può essere intenzionalmente privato della vita, salvo che in esecuzione di una sentenza capitale pronunciata da un tribunale, nel caso in cui il delitto è punito dalla legge con tale pena.

2. La morte non si considera inflitta in violazione di questo articolo quando risulta da un ricorso alla forza resosi assolutamente necessario:

a. per assicurare la difesa di ogni persona dalla violenza illegale; $b$. per eseguire un arresto regolare o per impedire l'evasione di una persona legalmente detenuta;

c. per reprimere, in modo conforme alla legge, una sommossa o un'insurrezione"

50 Cfr. Sentenze Grande Camera del 17 gennaio 2002, causa 32967/96, Calvelli e Ciglio c. Italia; del 24 ottobre 2002, causa 37703/97, Mastromatteo c. Italia; del 8 luglio 2004, causa 53924/00, Vo c. Francia; del 12 maggio 2005, causa 46221/99, Ocalan c. Turchia.

51 C. ZANGHI', "La protezione internazionale dei diritti dell'uomo", Torino, 2006, p. 255 dell'individuo ${ }^{52}$, nella tutela dell'individuo la cui vita sia minacciata da comportamenti criminosi di terzi ${ }^{53}$ o dal rischio di una malattia in grado di cagionare la morte ${ }^{54}$ e nella prevenzione efficace e dissuasiva del pregiudizio alla vita ${ }^{55}$.

Dall'analisi precedentemente condotta emerge che, nei casi considerati, gli obblighi derivanti dalla norma in oggetto non siano stati soddisfatti motivo per il quale si tende a ritenere come assolutamente necessaria e pertinente la richiesta e conseguente adozione di misure provvisorie.

\section{L'art. 3: Divieto della tortura}

I diritti garantiti in questa disposizione si sono prestati, forse più di altri, ad interpretazioni di tipo evolutivo ${ }^{56}$.

È possibile evincere dalla giurisprudenza come l'art. 3 sia venuto in rilievo per tutelare il diritto alla "salute"57 e quello al "riconoscimento di pensioni sociali" ${ }^{58}$.

In merito a quest'ultimo aspetto la Corte ha precisato che ${ }^{59}$ : "(..) In the modern democratic State many individuals are, for all or part of their lives, completely dependent for survival on social

52 Cfr. Sentenze della Corte Europea dei Diritti dell'Uomo del 29 aprile 2002, causa 2346/02, Pretty c. Regno Unito; del 17 gennaio 2002, causa 32967/96, Calvelli e Ciglio c. Italia.

53 Cfr. Sentenze della Corte Europea dei Diritti dell'Uomo del 28 ottobre 1998, causa 23452/94, Osman c. Regno Unito, par. 115; del 28 marzo 2000, causa 22492/93, Kilic c. Turchia, par. 62 e 67; del 29 aprile 2002, causa 2346/02, Pretty c. Regno Unito.

54 Cfr. Sentenze della Corte Europea dei Diritti dell'Uomo del 2 settembre 1998, causa 22495/93, Yasa c. Turchia, par. 92 - 108; del 28 ottobre 1998, causa 23452/94, Osman c. Regno Unito, par. 115 - 122; del 20 dicembre 2004, causa, Makaratzis c. Grecia, cap. 50 .

55 Cfr. mutatis mutandis, Osman c. Regno Unito, del 28 ottobre 1998; Kilic c. Turchia, del 28 marzo 2000.

56 C. ZANGHI', "La protezione internazionale dei diritti dell'uomo", Torino, 2006, p. 247.

57 Cfr. Sentenze della Corte Europea dei Diritti dell'Uomo del 23 aprile 2002, causa 56869/00, Larioshina c. Russia; del 18 giugno 2009, causa 45603/05, Budina c. Russia; del 24 aprile 2012, causa 25446/06, Yordanova e altri c. Bulgaria; del 23 giugno 2014, causa n. 46043/14, Pierre Lambert e altri c. Francia.

58 Cfr. Convegno annuale dell'Associazione "Gruppo di Pisa": "I diritti sociali: dal riconoscimento alla garanzia. Il ruolo della giurisprudenza", Trapani, 8-9 giugno 2012.

59 Cfr. Sentenze della Corte Europea dei Diritti dell'Uomo del 15 settembre 2009, causa 10373/05, Moskal c. Polonia; del 30 settembre 2003, causa 40892/98, Koua Poirrez c. Francia; del 9 luglio 2009, causa 46368/06, Zeibek c. Grecia; del 18 febbraio 2009, causa 55707/00, Andrejeva c. Lettonia; del 15 marzo 2011, causa 43134/05, G.N. c. Italia. 
security and welfare benefits. Many domestic legal systems recognise that such individuals require a degree of certainty and security, and provide for benefits to be paid - subject to the fulfilment of the conditions of eligibility - as of right. Where an individual has an assertable right under domestic law to a welfare benefit, the importance of that interest should also be reflected by holding Article 1 of Protocol No. 1 to be applicable.

The mere fact that a property right is subject to revocation in certain circumstances does not prevent it from being a "possession" within the meaning of Article 1 of Protocol No. 1, at least until it is revoked.

On the other hand where a legal entitlement to the economic benefit at issue is subject to a condition, a conditional claim which lapses as a result of the non-fulfilment of the condition cannot be considered to amount to "possessions" for the purposes of Article 1 of Protocol No. 1.(..)" 60 .

Ciò rileva in maniera determinante per la questione che qui si sta trattando.

Nei casi oggetto d'esame, infatti, il mancato riconoscimento dei "benefici" in favore delle vittime costituisce una violazione degli artt. 3 della Convenzione e 1 del Protocollo n.1.

\section{L'art. 8: Diritto al rispetto della vita privata e familiare ${ }^{61}$}

I diritti garantiti in tale disposizione hanno un'ampia interpretazione. Le nozioni di "vita privata e familiare" si sono, nel corso del tempo, giuridicamente evolute ed estese.

Nella "vita privata" si tende, infatti, a far rientrare anche la salute ${ }^{62}$, i comportamenti

60 Cfr. Sentenza della Corte Europea dei Diritti dell'Uomo del 15 settembre 2009, causa 10373/05, Moskal c. Polonia, \$§ 39 - 40.

61 Art. 8: "1. Ogni persona ha diritto al rispetto della sua vita privata e familiare, del suo domicilio e della sua corrispondenza. 2. Non può esservi ingerenza di un'autorità pubblica nell'esercizio di tale diritto a meno che tale ingerenza sia prevista dalla legge e costituisca una misura che, in una società democratica è necessaria per la sicurezza nazionale, per la pubblica sicurezza, per il benessere economico del Paese, per la difesa dell'ordine e per la prevenzione dei reati, per la protezione della salute o della morale, o per la protezione dei diritti e delle libertà altrui".

62 Cfr. Sentenza della Grande Camera del 19 ottobre 2005, causa 32555/96, Roche c. Regno Unito, par. 155 ss. sessuali, l'identità della persona, il nome ${ }^{63}$ e la riservatezza dei dati sanitari ${ }^{64}$.

Altrettanto interessante è l'interpretazione data all'accezione di "vita familiare" nella quale vengono ricompresi i diritti parentali ${ }^{65}$, la corrispondenza e il domicilio personale ${ }^{66}$ e professionale ${ }^{67}$.

Proprio in merito a quest'ultimo aspetto la Corte ha messo in luce la possibilità di interpretare l'art. 8 come «comprendente, in determinate circostanze, il diritto di una società al rispetto della sua sede sociale, la sua agenzia o i suoi locali professionali ${ }^{68} »$ ed ha inoltre evidenziato che «pregiudizi gravi all'ambiente possono intaccare il benessere di una persona e privarla del godimento del suo domicilio così da nuocere alla sua vita privata e familiare ${ }^{69}$.

Pertanto, nei casi di gravi rischi per la salute si è propeso per una soluzione di giusto bilanciamento fra le vittime e gli interessi concorrenti della comunità in correlazione con un ruolo attivo da parte dello Stato il quale è invitato ad intervenire "per tutelare la salute dei cittadini da qualsiasi rischio che possa discendere anche dall'industria privata» ${ }^{70}$.

L'art. 8 sembrerebbe essere pertanto un prolungamento ed un completamento del diritto alla salute sancito all'art. 3 .

L'esposizione a sostanze nocive (quali amianto, uranio impoverito, etc.) tenderebbe a negare gli obblighi che sono in capo allo Stato. Nei casi considerati le vittime sono obbligate a continuare

63 Cfr. Sentenze della Corte Europea dei Diritti dell'Uomo del 22 febbraio 1994, causa 16213/90, Burghartz c. Svizzera.

64 Cfr. Sentenza della Corte Europea dei Diritti dell'Uomo del 25 febbraio 1997, causa 22009/93, Z. c. Finlandia.

65 Cfr. Sentenza della Corte Europea dei Diritti dell'Uomo del 7 agosto 1996, causa 17283/90, Johansen c. Norvegia.

66 Cfr. Sentenza della Corte Europea dei Diritti dell'Uomo del 18 luglio 2006, causa 28867/03, Keegan c. Regno Unito, par. 29 ss.

67 Cfr. Sentenza della Corte Europea dei Diritti dell'Uomo del 25 febbraio 2003, causa 51772/99, Roemen e Schmit c. Lussemburgo, Raccolta, 2003 - IV, par. 64.

68 C. ZANGHI', "La protezione internazionale dei diritti dell'uomo", Torino, 2006, p. 271

69 Cfr. Sentenza della Corte Europea dei Diritti dell'Uomo del 9 dicembre 1994, causa 16798/90, Lopez Ostra c. Spagna.

70 Cfr. Sentenza della Grande Camera del 8 luglio 2003, causa 36022/97, Hatton c. Regno Unito, Raccolta, 2003 - VIII, PAR. 116 ss.; Sentenza della Corte Europea dei Diritti dell'Uomo del 9 giugno 2005, causa 55723/00, Fadeïeva c. Russia, parr. $104-$ 105 e 116 ss. 
a lavorare in esposizione a sostanze nocive cosa questa che si traduce nella promozione e accelerazione delle patologie "asbesto correlate"71.

Grave è da ritenersi la condotta dello Stato nella misura in cui obblighi le presunte vittime, lavoratori che, per diritto, dovrebbero essere collocati in pensione, a continuare a lavorare in condizioni di accresciuta pericolosità e di rischio per la salute.

Si tende a ritenere pertanto che, anche in questo caso, lo Stato abbia disatteso ai propri obblighi e creato una fattispecie idonea alla richiesta di misure provvisorie.

La potenziale inosservanza delle misure provvisorie da parte dello Stato e la giustificata invocazione di violazione dell'art. 34 della Convenzione da parte della Corte $^{72}$

L'art. 34 è una norma di straordinaria portata che conferisce al ricorrente un diritto di natura processuale.

L'individuo che sia "legittimato ad agire" può ricorrere liberamente contro qualsiasi Parte contraente. La legittimazione ad agire gli viene direttamente attribuita dalla precisa condizione di potersi pretendere vittima di una violazione.

Tale disposizione, il cui richiamo in termini di violazione di misure provvisorie ha da sempre ricevuto numerose critiche da parte della dottrina che vi ravvedrebbe un semplice rapporto di subordinarietà dell'elemento sostanziale a quello procedurale $^{73}$, tenderebbe ad essere violata nel caso specifico proprio a causa della morte del

71 E. BONANNI, "Lo Stato dimentica l'amianto killer. Diritti negati. Ricorso a Strasburgo", Firenze, 2009, p. 169. Cfr. Cass. IV Sez. Pen., del 2008, causa 42128.

72 Art. 34: "1. La Corte può essere investita di una domanda fatta pervenire da ogni persona fisica, ogni organizzazione non governativa o gruppo di privati che pretenda d'essere vittima di una violazione da parte di una delle Alte Parti contraenti dei diritti riconosciuti nella Convenzione o nei suoi protocolli. Le Alte Parti contraenti si impegnano a non ostacolare con alcuna misura l'effettivo esercizio di tale diritto.

73 In merito a questo aspetto si tende invece a sostenere che: L'individuazione da parte della Corte dello stretto legame fra l'articolo 39 del Regolamento e l'articolo 34 della Convenzione contribuisce a rafforzare la convinzione che vi sia da parte di questa l'intenzione di trasferire "per riflesso" all'elemento sostanziale e alla materia cautelare in generale l'importanza che, da sempre, viene attribuita nel sistema convenzionale $e$ che è desumibile anche dalla giurisprudenza, all'oggetto della procedura. (Tesi dottorale: "Le misure provvisorie secondo la Cedu e nella giurisprudenza della Corte di Strasburgo"). ricorrente che andrebbe a vanificare il ricorso privandolo del suo "oggetto".

Pertanto, non sembra improprio concludere che anche l'aspetto procedurale sia da considerarsi violato.

\section{CONCLUSIONI}

Esaminati i differenti argomenti è possibile trarre alcune conclusioni.

Anzitutto si impone una precisazione di carattere terminologico atta ad agevolare la Corte ad esaminare solo i ricorsi che presentino determinate caratteristiche.

I ricorrenti potrebbero riportare in prima pagina la dicitura: "lavoratori con patologie terminali da esposizione a sostanze nocive".

Un'ulteriore considerazione che si impone è quella, per altro già formulata, relativa alla evidente pertinenza di questi casi con l'ambito di applicazione della tutela cautelare. Le violazioni riscontrate, "sostanziali" (artt. 2, 3 e 8 CEDU), e, "procedurali" (art. 34 CEDU), rientrano fra quelle indicate dalla Corte stessa ${ }^{74}$ come idonee affinché la presunta vittima sia "legittimata" ad avanzare richiesta di adozione di misure provvisorie.

Come già affermato le soluzioni interne si presentano a tal punto inefficaci ed inefficienti da rendere spesso vano l'esercizio del diritto di ricorso da parte delle vittime.

Ciò tende a rendere assolutamente necessaria l'esistenza di una procedura da cui possa scaturire una decisione tempestiva capace di conferire ai potenziali ricorrenti la tutela di quei diritti attualmente negati.

A questo riguardo occorre far presente che in alcun modo si tenderebbe ad intaccare il principio che sottende ai rapporti fra ordinamenti. L'adozione della misura provvisoria interverrebbe, infatti, al solo scopo di creare una situazione temporanea in attesa del giudizio interno. Tale soluzione sembrerebbe essere l'unica in grado di offrire alle vittime quel ristoro "immediato" "essenziale" a sollevarle, in quanto già gravemente malate, dall'obbligo di continuare a lavorare in esposizione a sostanze che tendono ad accelerare il decorso della malattia.

74 Cfr. Sentenza della Corte europea dei Diritti dell'Uomo, op. cit., Mamatkulov et Askarov c. Turchia, par. 104. 
Viepiù che, in questo modo, si tenderebbe ad arginare, seppur non totalmente ma almeno parzialmente, la questione sollevata in più sedi ${ }^{75}$, dell'accertamento del fatto ${ }^{76}$.

Richiamate queste opportune osservazioni sembra opportuno concludere rammentando che: "Nessuna delle disposizioni della presente Convenzione può essere interpretata come recante pregiudizio o limitazione ai diritti dell'uomo e alle libertà fondamentali che possano essere riconosciuti in base a leggi di qualunque Stato contraente o da altri accordi internazionali di cui tale Stato sia parte". La norma, "pur riconoscendo la supremazia dei diritti già vigenti o di quelli che potrebbero essere successivamente riconosciuti, determina, in ogni singolo caso, l'applicazione della norma più favorevole all'individuo"77.
Inoltre la più recente giurisprudenza a cui si è fatto riferimento nel corso della trattazione ${ }^{78}$ sembrerebbe essere intervenuta proprio a sostegno di tale teoria.

Pertanto, si tende a concludere che, nei casi che si sono fatti oggetto di studio, il sistema più favorevole all'individuo sembrerebbe essere proprio quello di tutela cautelare in seno alla Corte di Strasburgo.
75 FABIANI E. BONANNI, "Il danno da amianto. Profili risarcitori e tutela medico - legale", Milano, 2013.

76 Vi si sollevano a tal proposito sempre numerosi problemi dato che alla lunga latenza delle patologie si lega il profondo mutamento della situazione fattuale in cui la vittima ha prestato la propria attività.

77 C. ZANGHI', "La protezione internazionale dei diritti dell'uomo", Torino, 2006, p. 168.
78 Cfr. Corte Europea dei Diritti dell'Uomo, del 23 giugno 2014 causa n. 46043/14, Pierre Lambert e altri c. Francia e Corte di Cassazione, IV^ $V^{\wedge}$ se. pen., 08.05.2014, n. 18933. 


\section{BIBLIOGRAFÍA}

- CANÇADO TRINDADE, "Les mesures provisoires devant la Cour interaméricaine des droits de l'homme", in COHEN-JONATHAN e FLAUSS, Mesures conservatoires et droits fondamentaux, Bruxelles, 2005, pp. 148-149.

- CHIOVENDA, Istituzioni di diritto processuale civile, Napoli, 1933.

- COHEN - JONATHAN, De l'effet juridique des "mesures provisoires" dans certaines circonstances et de l'efficacité du droit de recours individuel: à propos de l'arrêt de la Cour de Strasbourg Cruz Varas du 20 mars 1991, in RUDH, 1991, p. 205.

- CORNU (dir.),Vocabulaire juridique, 6 ed., Paris, 2004.

- Note d'information sur la jurisprudence de la Cour, n. 176, luglio 2014.

- DE SALVIA, Sistema europeo e sistemi nazionali di protezione dei diritti dell'uomo: subordinazione, sussidiarietà?, in RIDU, 1994, p. 24.

- DE SALVIA, Ricorrere a Strasburgo: presupposti e procedura, Milano, Giuffrè, 2011.

- FLAUSS, Mesures provisoires et droits fondamentaux, COHEN-JONATHAN e FLAUSS (a cura di), Droit et Justice, Bruylant, 2005, p. 199.

- GAETA, I provvedimenti cautelari nel sistema europeo di protezione dei diritti dell'uomo, in RID, 1996, p. 34.

- QUATTROCCHI, tesi dottorale, "Le misure provvisorie secondo la Cedu e nella giurisprudenza della Corte di Strasburgo".

- SACCUCCI, Le misure provvisorie nella protezione internazionale dei diritti umani, Torino, 2006.

- ZANGHÍ, La protezione internazionale dei diritti dell'uomo, Torino, 2006.

- Amianto

- Air Force Armament Laboratory, Morphological Characteristics of Particulate material Formed from High Velocity Impact of Depleted Uranium Projectiles with Armor Targets. Final Report for period October
1977 - October 1978; IARC, Monograph vo. 14, "Asbetos", 1977.

- Bonanni, Lo Stato dimentica l'amianto Killer, Firenze, 2009.

- Fabiani, Bonanni, "Il danno da amianto. Profili risarcitori e tutela medico - legale", Milano, 2013.

- Governa, Amati, Fontana et al., Role of the iron in absestos - body - induced oxidant radical generation, in Toxicol Environ Healt part A, 1999; 58: pp. 279 - 87.

- Gustavsson et al., "Occupational exposures and squamous cell carinoma of the oral cavity, pharinx, larynx and oesophagus: a case - control study in Sweden", in Occup. Environ med. 55, pp. 393 - 400, 1998.

- Documentazione amianto

- Gazzetta Ufficiale n. 87 del 13 aprile 1992

- Gazzetta Ufficiale n. 229 del 2 ottobre 2003

- Gazzetta Ufficiale n. 101 del 30 aprile 2008

- Legge 4 agosto 1993, n. 271

- Legge 27 marzo 1992 n. 257: norme relative alla cessazione dell'impiego dell'amianto.

- Relazione Giudice del Lavoro Tribunale di Ravenna Dott. Roberto Riverso per incontro CSM sulla formazione dei magistrati del 26.11.2008 "Il rischio amianto: le controversie in materia di maggiorazione contributiva".

\section{GIURISPRUDENZA CITATA}

- 6 ottobre 1977, causa 7317/75, Lynas c. Svizzera

- 7 luglio 1989, causa 14038/88, Soering c. Regno Unito

- 20 marzo 1991, causa 15576/89, Cruz Varas e altri c. Svezia

- 30 ottobre 1991, causa 13163/87, 13164/87, 13165/87, Vilvarajah e altri c. Regno-Unito

- 2 maggio 1997, causa 30240/96, D. c. Regno Unito

- 11 luglio 2000, causa 40035/98, Jabari c. Turchia

- 5 febbraio 2002, causa 51564/99, Conka e altri c. Belgio 
- 8 luglio 2004, causa 48787/99, Ilascu e altri c. Moldavia e Russia

- 4 febbraio 2005, causa46827/99 e 46951/99, Mamatkulove Askarov c. Turchia

- 12 aprile 2005, causa 36378/02, Chamaïev e altri c. Georgia e Russia

- 10 novembre 2005, causa 26050/04, Gurbuz c. Turchia

- 10 novembre 2005, causa 24040/04, Kuruçay c. Turchia

- 10 novembre 2005, causa 8062/04, Sinan Eren c. Turchia

- 10 novembre 2005, causa 22913/04, Tekin Yildiz c. Turchia

- 10 novembre 2005, causa 7454/04, Uyan c. Turchia

- 10 agosto 2006 , causa 24668/03, Olaechea Cahuas c. Spagna

- 26 aprile 2007, causa 25389/05 Gebremedhin c. Francia

- 20 settembre 2007, causa 45223/05, Sultani c. Francia

- 28 febbraio 2008, causa 37201/06, Saadi c. Italia

- 27 marzo 2008, causa 44009/05, Chtoukatourov c. Russia

- 27 marzo 2008, causa 28320/02, Guidi c. Italia

- 22 dicembre 2008, causa 46468/06, Aleksanyan c. Russia

- 24 febbraio 2009, causa 246/07, Ben Khemais c. Italia

- 10 marzo 2009, causa 39806/05, Paladi c. Moldavia

- 18 giugno 2009, causa 45603/05, Budina c. Russia

- 22 settembre 2009, causa 30471/08, Abdolkhani e Kariminia c. Turchia

- 3 dicembre 2009, causa 19576/08, Daoudi c. Francia

- 2 marzo 2010, causa 61498/08, Al Saadoon e Mufdhi c. Regno Unito

- 1 aprile 2010, causa 24268/08, Klein c. Russia

- 13 aprile 2010, causa 50163/08, Trabelsi c. Italia
- 3 giugno 2010, causa 52812/07, Kamaliyevy c. Russia

- 21 gennaio 2011, causa 30696/09, M.S.S. c. Belgio e Grecia

- 5 aprile 2011, causa 25716/09, Toumi c. Italia

- 20 dicembre 2011, causa 10486/10, Yoh Ekale c. Belgio

- 10 gennaio 2012, causa 66697/10, Tamara Suzi c. Finlandia

- 17 gennaio 2012, causa 8139/09, Othman (Abou Qatada) c. Regno Unito

- 24 gennaio 2012, causa 26494/09, Ahmed Ali c. Paesi Bassi e Grecia

- 10 aprile 2012, causa 24027/07, 11949/08, 36742/08, 66911/09 e 67354/09, Babar Ahmad e altri c. Regno Unito

- 8 gennaio 2013, causa 10079/06, Jashi c. Georgia

- 23 giugno 2014, causa n. 46043/14, Pierre Lambert e altri c. Francia

- Amianto

- 12 novembre 2008, causa 42128, Sentenza della Corte di Cassazione IV Sez. Pen.

- Cassazione 21682/2004

- Cassazione Sezione Lavoro 16119/2005

- Cassazione 16119/2005

- Cassazione Sezione Lavoro n. 21256/2004; $2456 / 2005 ; 2587 / 2005 ; 1392 / 2005,2582$ 83 85/2005; $16117 / 2005$

- Sentenze del Tribunale di Terni confermate in Cassazione: 22432/2004; 22433/2004; 22435/2004; 22446/2004; 377/2004; 2243/2004; 22519/2004; 22541/2004; 22831/2004; 23524/2004; 21929 - 2031 32/2004; 21889 - 90 - 91/2004; 1980/2004; $4557 / 2004 ; 4550 / 2004$; 2415/2004; $1969 / 2004 ; 4665 / 2004 ; 3463 / 2004$; 2416/2004; 2408/2004; 2475/2004; 1974/2004; $22313-14-15-16-17-$ 18/2004; 22300/2004; $21864-65-66-67$ - 68/2004; 396/2004; 320/2004; 22156 57 - 58/2004; 21861/2004; 21991/2004; 22301 - 02 - 03 - 04/2004; 22079 - 80/2004; 20465/2004; 10673/2004; 21223/2004; 21330 - 31/2004; 21445/2004.

- Sentenza della Cassazione Sezione Lavoro n. $16119 / 2005$ 